\title{
Exposição ao benzeno em trabalhadores de postos de gasolina: Uma revisão integrativa da literatura
}

\author{
Exposure to benzene in gas station workers: An integrative literature review \\ Exposición al benceno en trabajadores de gasolineras: Una revisión integradora de la literatura
}

Recebido: 30/04/2021 | Revisado: 06/05/2021 | Aceito: 10/05/2021 | Publicado: 23/05/2021

\author{
Viviane Monteiro da Silva \\ ORCID: https://orcid.org/0000-0002-5136-2402 \\ Instituto Evandro Chagas, Brasil \\ E-mail: vivianemonteiro2outlook.com \\ Renata Chaves Medeiros \\ ORCID: https://orcid.org/0000-0002-1973-2063 \\ Escola Superior da Amazônia, Brasil \\ E-mail: chavesrenata39@gmail.com \\ Cristina do Socorro Prestes Modesto \\ ORCID: https://orcid.org/0000-0002-0538-6710 \\ Escola Superior da Amazônia, Brasil \\ E-mail: cristina.modesto.1966@gmail.com \\ Juan Gonzalo Bardález Rivera \\ ORCID: https://orcid.org/0000-0003-1737-6947 \\ Escola Superior da Amazônia, Brasil \\ E-mail: jgrivera@bol.com.br
}

\begin{abstract}
Resumo
A exposição ao benzeno gera prejuízos à saúde, fato que o tornou alvo da atenção dos órgãos de vigilância em saúde em todo o mundo. Este composto é capaz de causar danos hematológicos, genotóxicos e desenvolvimento de cânceres. O objetivo deste estudo é realizar o levantamento bibliográfico acerca dos efeitos tóxicos da exposição ao benzeno em trabalhadores de postos de gasolina. Trata-se de uma revisão integrativa da literatura com abordagem qualitativa. Foi realizado levantamento bibliográfico nas bases de dados Scielo, LILACS, LA Referência e PubMed, no período de agosto de 2010 a dezembro 2020, nos idiomas português, inglês e espanhol. A análise das publicações demonstrou que o biomarcadore mais adequado para o monitoramento da exposição ocupacional ao benzeno é o Ácido fenilmercaptúrico. As alterações hematológicas e os efeitos genotóxicos também são frequentemente encontrados em indivíduos expostos e são úteis como biomarcadores de efeito. Conclui-se que os trabalhadores de postos de revenda de combustíveis estão suscetíveis a diversos danos à saúde em decorrência da exposição ao benzeno, e que a legislação trabalhista precisa ser atualizada a fim de minimizar estes danos.
\end{abstract}

Palavras-chave: Exposição ocupacional; Benzeno; Biomarcadores; Genotoxicidade.

\begin{abstract}
Exposure to benzene causes damage to health, a fact that has made it a target of the attention of health surveillance agencies around the world. This compound is capable of causing damage hematological, genotoxic and cancer development. The purpose of this study is to carry out the bibliographic survey about the toxic effects of exposure to benzene in workers of gas station. This is an integrative literature review with qualitative approach. Was performed bibliographic survey in the Scielo, LILACS, LA Reference and PubMed, from August 2010 to December 2020, in Portuguese, English and Spanish. Analysis of the publications showed that the most appropriate biomarker for monitoring occupational exposure to benzene is the phenylmercapturic acid. Hematological changes and effects genotoxic substances are also often found in exposed individuals and are useful as effect biomarkers. It is concluded that the workers of resale posts of fuels are susceptible to a variety of health hazards as a result of exposure to benzene, and that labor legislation needs to be updated in order to minimize these damage.
\end{abstract}

Keywords: Occupational exposure; Benzene; Biomarkers; Genotoxicity.

\section{Resumen}

La exposición al benceno causa daños a la salud, hecho que lo ha convertido en el objetivo de atención de las agencias de vigilancia de la salud en todo el mundo. Este compuesto es capaz de provocar daños hematológicos, genotóxicos y el desarrollo de cánceres. El objetivo de este estudio es realizar un relevamiento bibliográfico sobre los efectos tóxicos de la exposición al benceno en trabajadores de gasolineras. Es una revisión integradora de la literatura con un enfoque cualitativo. Se realizó un relevamiento bibliográfico en las bases de datos Scielo, LILACS, LA Referência y PubMed, de agosto de 2010 a diciembre de 2020, en portugués, inglés y español. El análisis de las publicaciones mostró que el 
biomarcador más adecuado para monitorear la exposición ocupacional al benceno es el ácido fenilmercaptúrico. Los cambios hematológicos y los efectos genotóxicos también se encuentran con frecuencia en individuos expuestos y son útiles como biomarcadores de efectos. Se concluye que los trabajadores de las estaciones de reventa de combustible son susceptibles a varios daños a la salud como consecuencia de la exposición al benceno, y que la legislación laboral debe actualizarse para minimizar estos daños.

Palabras clave: Exposición ocupacional; Benceno; Biomarcadores; Genotoxicidad.

\section{Introdução}

O benzeno é um hidrocarboneto aromático, de fórmula C6H6, que se apresenta como um líquido incolor, volátil, altamente inflamável, explosivo, solúvel em solventes orgânicos e que possui alto grau de toxicidade para seres humanos. A Agência Internacional de Pesquisa em Câncer (IARC), desde 1982, reconhece o seu potencial carcinogênico. O benzeno é encontrado em diversos meios e produtos como: petróleo, carvão mineral, condensado de gás natural, nos processos de síntese química e na produção de aço (Correa \& Santana, 2016; Nogueira, 2016; Campos et al., 2017).

A exposição ao benzeno gera prejuízos a saúde, fato que o tornou alvo da atenção dos órgãos de vigilância em saúde em todo o mundo. De acordo, com os critérios do Programa de Segurança Química das Nações Unidas, o benzeno é classificado como a quinta substância de maior risco a saúde humana. Este composto é capaz de causar uma série de efeitos tóxicos ao organismo, o que inclui danos hematológicos, genotóxicos e mutagênicos (Moura-Correa et al., 2014; Valente et al., 2017).

Na década de 90 foi celebrado o Acordo Nacional do Benzeno (ANB) que culminou com a criação da Comissão Nacional Permanente do Benzeno. Esta comissão é responsável por gerir a efetivação do ANB, além de definir atribuições e procedimentos para limitar o uso e circulação do benzeno nas empresas que trabalham com produção, transporte, armazenamento e manipulação do mesmo e suas misturas líquidas que contenham um por cento (1\%) ou mais em volume, exceto empresas do ramo de combustíveis (Corrêa, 2014).

O contato com este toxicante expõe estes trabalhadores à diversos riscos a saúde, que podem variar desde uma dermatite até leucopenia, leucemia e alterações cromossômicas. A exposição desta população ocorre por longos períodos e por diversas vias, principalmente a via respiratória, por meio da inalação de seus vapores. A exposição ocorre de forma crônica, na qual, a suscetibilidade aos seus efeitos tóxicos é maior, logo, é imprescindível que se faça a monitorização biológica da saúde destes trabalhadores (D’Alascio et al., 2014; Correa \& Santana, 2016).

O objetivo deste estudo é realizar o levantamento bibliográfico acerca dos efeitos tóxicos da exposição ao benzeno em trabalhadores de postos de gasolina.

\section{Metodologia}

Trata-se de uma revisão integrativa da literatura com abordagem qualitativa. Este tipo de pesquisa possibilita análise e síntese de pesquisas relevantes sobre a temática, além de apontar lacunas a serem sanadas através da realização de novas pesquisas. A revisão integrativa da literatura é uma ferramenta valiosa para a saúde baseada em evidências pois, permite a elaboração de conclusões sobre áreas de estudo específicas a partir da condensação de diversos estudos científicos (Mendes et al., 2008; Landim et al., 2015).

As bases de dados utilizadas para a busca de publicações foram: Scielo (Scientific Electronic Library Online), LILACS (Literatura Latino-Americana e do Caribe em Ciências da Saúde), LA Referência (Rede Federada de Repositórios Institucionais de Publicações Científicas) e PubMed. Os descritores utilizados para a busca das publicações foram "exposição ocupacional", "benzeno", "biomarcadores" e "genotoxicidade". 
Foram utilizadas duas combinações dos descritores, a fim de obter artigos independentes sobre biomarcadores e genotoxicidade. A primeira combinação utilizada foi: "benzeno" AND "exposição ocupacional” AND "biomarcadores". A segunda combinação utilizada foi "benzeno" AND "exposição ocupacional" AND "genotoxicidade". Os descritores selecionados são indexados de acordo com o Portal de Descritores em Ciências da Saúde (DECS). Os critérios de inclusão para a pesquisa de publicações foram: texto completo disponível, publicados nos idiomas português, inglês e espanhol, e que tenham sido publicadas no período de agosto de 2010 a dezembro de 2020.

\section{Resultados e Discussão}

O levantamento bibliográfico utilizando-se a combinação 1 de descritores (benzeno AND exposição ocupacional AND biomarcadores), aplicando-se os critérios de inclusão e exclusão, resultou em 5 publicações na base de dados LILACS, 6 na base Scielo, 14 na base LA referência, e 58 na base Pubmed. Com a combinação 2 (benzeno AND exposição ocupacional AND genotoxicidade), aplicando-se os critérios de inclusão e exclusão, obteve-se 5 publicações na base de dados LILACS, 3 na base Scielo, 7 na base LA Referência, e 19 na base Pubmed.

Observou-se repetição de um grande quantitativo de artigos entre as bases de dados. Após exclusão dos artigos repetidos entre as duas combinações, foi realizada a leitura dos resumos dos estudos para selecionar os que se adequam ao tema desta pesquisa, ou seja: estudos que tratem sobre os biomarcadores e/ou genotoxicidade relacionados à exposição ocupacional ao benzeno. Esta seleção reduziu o total da amostra para 26 publicações, listadas no Quadro 1. A análise dos artigos selecionados possibilitou a extração de informações pertinentes aos objetivos desta revisão, as quais serão discutidas nos capítulos subsequentes.

Quadro 1 - Amostra final de artigos resultantes do levantamento bibliográfico.

\begin{tabular}{|c|c|}
\hline Título do estudo & $\begin{array}{l}\text { Autores, ano de } \\
\text { publicação }\end{array}$ \\
\hline $\begin{array}{l}\text { Avaliação da exposição ao benzeno, tolueno, etilbenzeno e xilenos (btex) em trabalhadores expostos } \\
\text { ocupacionalmente, no município do Rio de Janeiro }\end{array}$ & Figueiredo, 2018 \\
\hline $\begin{array}{l}\text { Proteomics analysis identified serum biomarkers for occupational benzene exposure and chronic } \\
\text { benzene poisoning }\end{array}$ & Li et al., 2019 \\
\hline $\begin{array}{l}\text { Avaliação dos efeitos genotóxicos da exposição ocupacional em frentistas atuantes em postos de } \\
\text { gasolina no município de Santarém, Pará }\end{array}$ & Maciel et al., 2019 \\
\hline $\begin{array}{l}\text { Effect of Benzene Exposure on the Urinary Biomarkers of Nucleic Acid Oxidation in Two Cohorts } \\
\text { of Gasoline Pump Attendants }\end{array}$ & Carrieri et al., 2019 \\
\hline $\begin{array}{l}\text { Hematological and hepatic alterations in nonsmoking residents exposed to benzene following a } \\
\text { flaring incident at the British petroleum plant in Texas City }\end{array}$ & D'andrea \& Reddy, 2014 \\
\hline $\begin{array}{l}\text { Avaliação da exposição ocupacional ao benzeno em trabalhadores frentistas e analistas de } \\
\text { combustíveis utilizando o Teste Cometa como biomarcador de genotoxicidade }\end{array}$ & Campos et al., 2017 \\
\hline Aspectos toxicológicos do benzeno, biomarcadores de exposição e conflitos de interesses & Santos et al., 2017 \\
\hline $\begin{array}{l}\text { Benzene Exposure and Biomarkers in Alveolar Air and Urine Among Deck Crews on Tankers } \\
\text { Transporting Gasoline }\end{array}$ & Forsell et al., 2019 \\
\hline $\begin{array}{l}\text { Changes in Oxidative Stress Biomarker and Gene Expression Levels in Workers Exposed to Volatile } \\
\text { Organic Compounds }\end{array}$ & Kim et al., 2011 \\
\hline $\begin{array}{l}\text { Avaliação ambiental de BTEX (benzeno, tolueno, etilbenzeno, xilenos) e biomarcadores de } \\
\text { genotoxicidade em trabalhadores de postos de combustíveis }\end{array}$ & Amaral et al., 2017 \\
\hline Genotoxic effects of occupational exposure to benzene in gasoline station workers & Salem et al., 2018 \\
\hline $\begin{array}{l}\text { Níveis de trans, trans-mucônico na urina como biomarcador de exposição ao benzeno e alterações } \\
\text { hematológicas na população do bairro Piquiá de Cima, Açailândia - MA }\end{array}$ & Araújo, 2015 \\
\hline
\end{tabular}




\begin{tabular}{|c|c|}
\hline $\begin{array}{l}\text { Teste cometa: validação do método e avaliação da exposição ocupacional ao benzeno presente na } \\
\text { gasolina através dos biomarcadores de exposição e genotoxicidade }\end{array}$ & Campos, 2013 \\
\hline $\begin{array}{l}\text { Estratégias analíticas com cromatografia e espectrometria de massas para biomonitorização da } \\
\text { exposição ao benzeno pela determinação do ácido } S \text {-fenilmercaptúrico urinário }\end{array}$ & Gonçalves et al., 2017 \\
\hline $\begin{array}{l}\text { Factors Affecting Urinary tt-Muconic Acid Detection among Benzene Exposed Workers at Gasoline } \\
\text { Stations }\end{array}$ & Chaiklieng et al., 2019 \\
\hline Renal and hepatotoxic alterations in adult mice on inhalation of specific mixture of organic solvents & Ketan et al., 2015 \\
\hline $\begin{array}{l}\text { Exposição ao benzeno em postos de revenda de combustíveis no Brasil: Rede de Vigilância em } \\
\text { Saúde do Trabalhador (VISAT ) }\end{array}$ & Moura-Corrêa, 2014 \\
\hline $\begin{array}{l}\text { Alteraciones hematol ógicas en trabajadores expuestos ocupacionalmente a mezcla de Benceno- } \\
\text { Tolueno-Xileno (BTX) en una fábrica de pinturas }\end{array}$ & Haro-García et al., 2012 \\
\hline $\begin{array}{l}\text { Adverse Health Effects of Benzene Exposure Among Children Following a Flaring Incident at the } \\
\text { British Petroleum Refinery in Texas City }\end{array}$ & D’andrea \& Reddy, 2014 \\
\hline $\begin{array}{l}\text { Evaluation of immunological, inflammatory, and oxidative stress biomarkers in gasoline station } \\
\text { attendants }\end{array}$ & Moro et al., 2019 \\
\hline $\begin{array}{l}\text { Classificação clínico-laboratorial para manejo clínico de trabalhadores expostos ao benzeno em } \\
\text { postos de revenda de combustíveis }\end{array}$ & Fonseca et al., 2017 \\
\hline $\begin{array}{l}\text { Environmental Assessment and Evaluation of Oxidative Stress and Genotoxicity Biomarkers Related } \\
\text { to Chronic Occupational Exposure to Benzene }\end{array}$ & Costa-Amaral, 2019 \\
\hline $\begin{array}{l}\text { Normas ocupacionais do benzeno: uma abordagem sobre o risco e exposição nos postos de revenda } \\
\text { de combustíveis }\end{array}$ & Mendes et al., 2017 \\
\hline $\begin{array}{l}\text { Sintomas relacionados à exposição ocupacional ao benzeno e hábitos ocupacionais em trabalhadores } \\
\text { de postos de revenda de combustíveis a varejo na região sul de Santa Catarina }\end{array}$ & D’alascio et al., 2013 \\
\hline Intoxicação ocupacional pelo benzeno: um assunto de saúde ambiental & Moreira \& Gomes, 2011 \\
\hline $\begin{array}{l}\text { Estudo da exposição ocupacional e ambiental ao benzeno em baixas concentrações sob a perspectiva } \\
\text { dos efeitos genotóxicos }\end{array}$ & Costa-Amaral, 2017 \\
\hline
\end{tabular}

Fonte: Autores.

\section{Indicadores bioquímicos de exposição ao benzeno}

No que se refere a sua absorção pelo organismo, sabe-se que a maior parte do benzeno inalado é eliminada pela expiração, a parte absorvida, cai na corrente sanguínea e se acumula principalmente em tecidos com alto teor de lipídios. Ess a absorção varia entre $10 \%$ a $50 \%$, dependendo da dose, do metabolismo e da quantidade de gordura presente no organismo (Khalade et al., 2010).

O percentual que permanece no organismo sofre transformações no fígado e medula óssea, após essas transformações, cerca de $1 \%$ desse composto é eliminado na urina na forma de metabólitos como o fenol, o catecol, hidroquinona, ácido transtrans mucônico (AttM) e ácido fenilmercaptúrico (AFM). Estes metabólitos são utilizados como biomarcadores de exposição no monitoramento da saúde de trabalhadores expostos. Recentemente o AFM tem sido recomendado pelas agências de saúde em virtude dos demais metabólitos sofrerem influência de outros contaminantes e até de hábitos alimentares (Pelallo-Martínez et al., 2014; Gonçalves et al., 2017; Santos et al., 2017; Costa-Amaral et al., 2019).

No estudo de Ibrahim et al (2014) o nível de AttM na urina dos trabalhadores expostos foi mais elevado que nos indivíduos não expostos, e particularmente elevado entre os fumantes. Araújo (2015) constatou níveis baixos de AttM na população estudada, mas esta estava sujeita a exposição ambiental ao benzeno, e não ocupacional. Figueiredo (2018) não encontrou correlação entre as concentrações de benzeno no ar e os níveis de AttM, sugerindo então que o AttM não é o biomarcador ideal para monitoramento da exposição ao benzeno por ser influenciado por outros fatores. Já Forsell (2018) 
encontrou correlação entre o benzeno no ar inalado e as concentrações de AttM em um estudo britânico com transportadores de tanques de combustíveis, em que os participantes não fumaram ao longo do dia da coleta.

Moura-Correa et al (2014) avaliou a exposição ao benzeno em trabalhadores de postos de revenda combustíveis em seis estados brasileiros, e constatou alterações nos marcadores de função hepática, renal e nos indicadores de dose interna (AttM e AFM).

Moro et al (2019) comparou um grupo de trabalhadores de postos de gasolina com um grupo controle e constatou níveis mais elevados de AttM no grupo exposto em relação ao não exposto, ainda que os valores médios do primeiro grupo estivessem abaixo do índice biológico de exposição estabelecido pela ACGIH (American Conference of Governamental Industrial Hygienists). No estudo de Chaiklieng et al (2019) 1/4 dos trabalhadores avaliados apresentaram AttM na urina, e cerca de 6 por cento destes, em valores acima dos preconizados pela ACGIH.

Carrieri et al (2019) avaliou os níveis de AFM em trabalhadores de postos de gasolina dividindo-os em dois grupos. O grupo 1 composto por trabalhadores com jornadas de 10-12h, e o grupo 2 por trabalhadores com jornadas de $8 \mathrm{~h}$. Os valores de AFM foram consideravelmente mais elevados entre indivíduos do grupo 1, tanto antes quanto após a jornada de trabalho. A possível explicação é que a jornada mais extensa pode resultar em acumulação dos metabólitos do benzeno.

Em um estudo transversal feito por Cintra (2016), investigou-se a exposição ao benzeno em trabalhadores de postos de gasolina. Os resultados encontrados mostram que, 7,1\% dos trabalhadores expostos apresentaram valores de AST alterados e com uma média igual a 56,8 U/l. Em relação a análise dos valores de ALT foi achado que, 24\% dos trabalhadores expostos apresentaram valores alterados, apresentando uma média igual a 65,08 U/1. A respeito dos achados bioquímicos sobre o GamaGT, observou-se que 24,3\% dos trabalhadores do grupo exposto apresentaram valores aumentados, com média de 111,07 U/1. $\mathrm{Na}$ análise bioquímica da lactato desidrogenase (LDH), foi visto que, 18,07\% dos trabalhadores do grupo exposto apresentaram valores elevados, com uma média de 521,9 U/l. Já na análise bioquímica dos valores de creatinina, observou-se que 2,2\% dos trabalhadores expostos apresentaram valores acima do valor de referência, com uma média de 1,6 mg/dl.

No estudo de Ketan et al (2015), foram avaliadas alterações renais e hepatotóxicas provocadas por inalação de uma mistura de solventes orgânicos (benzeno, tolueno e xileno) em camundongos. Nos animais expostos observou-se aumento significativo dos níveis de colesterol e creatinina, o que indica possível diminuição da renovação metabólica e acúmulo de seus produtos. Também foi identificado aumento significativo da atividade de ALT (alanina aminotransferase) e AST (aspartato aminotransferase), além de aumento dos níveis séricos dos hormônios tireoideanos T3 e T4, sugerindo hipertireoidismo transitório.

Os estudos de D’Andrea e Reddy (2014) avaliou seres humanos expostos e não expostos ao benzeno e corroborou achados semelhantes. Os níveis de creatinina, AST, ALT e ALP (fosfatase alcalina) séricas mostraram-se significativamente aumentados no grupo exposto, tanto em homens quanto mulheres. A elevação dos níveis séricos dessas enzimas pode estar relacionado a uma hiperprodução de enzimas por células hepáticas em resposta a estímulos por lesão ou morte celular no fígado. Os mecanismos exatos de produção exacerbada dessas enzimas em situações de exposição ao benzeno ainda não estão completamente esclarecidos.

\section{Indicadores hematológicos de exposição ao benzeno}

Os principais agravos relacionados ao benzeno estão ligados à hematotoxicidade e genotoxicidade decorrentes de sua metabolização. Os mecanismos de ação são complexos e ainda não estabelecidos por completo. A hematotoxicidade se manifesta principalmente pela diminuição das células sanguíneas, podendo resultar em diversas formas de queda no número dos elementos sanguíneos: anemia, leucopenia, plaquetopenia, ou das três séries em conjunto, na forma de pancitopenia. Estas alterações hematológicas, quando relacionadas ao benzeno, são consideradas um indicador precoce de leucemia mieloide 
aguda e síndrome mielodisplásica, embora não constituam uma etapa necessária para o desenvolvimento dessas doenças (Weisel, 2010; Kim et al., 2011; Manini et al., 2007).

O benzeno age, através de seus produtos de transformação, sobre a medula óssea, atingindo as células do sistema hematopoiético. A supressão da medula óssea (ou mielotoxicidade) pode causar redução do número de células sanguíneas primitivas e/ou provocar alterações estruturais ou citogenéticas, as quais têm como consequência a hipoprodução celular e/ou o surgimento de linhagens de células anormais (Arcuri et al, 2005, Li et al., 2019).

Desta maneira, a exposição crônica a baixas concentrações de benzeno pode produzir uma diminuição reversível nas contagens de células sanguíneas. No entanto, a exposição crônica a elevadas concentrações levaria a depressão irreversível da medula óssea, resultando em anemia, leucopenia, linfocitopenia e/ou trombocitopenia (Ruiz et al., 1994; Fonseca et al., 2017).

Os estudos de Moro e colaboradores (2019) mostram que a exposição ocupacional ao benzeno afeta glóbulos vermelhos, hematócrito e contagens de neutrófilos; no entanto, esses parâmetros permaneceram dentro dos valores de referência. Nesse sentido, as alterações no número de células sanguíneas não são especialmente sensíveis e não são indicadores dos primeiros efeitos do benzeno (Swaen et al., 2010).

Ray et al (2007), em seu estudo de exposição ao benzeno a partir de fontes veiculares e seus impactos na saúde realizado em Calcutá, Índia, investigaram a presença de alterações hematológicas em atendentes de postos de gasolina, mecânicos e num grupo de controles. Em comparação com os controles, os trabalhadores expostos ao benzeno apresentaram redução significativa na concentração de hemoglobina e na contagem de eritrócitos, linfócitos e plaquetas.

No Egito, Ibrahim et al (2014) investigou um grupo de homens e mulheres trabalhadores de uma fábrica de decoração de cerâmica, expostos ao benzeno. Foi observado que os trabalhadores apresentavam menor contagem de eritrócitos, leucócitos, plaquetas, menor concentração de hemoglobina e hematócrito quando comparado com um grupo de trabalhadores não expostos ao benzeno.

Os resultados do estudo de D'Andrea e Reddy (2014) demonstraram que indivíduos expostos ao benzeno apresentaram níveis mais altos de contagem de leucócitos $(7,7 \pm 2,2$ versus $6,8 \pm 1,7)$ e contagem de plaquetas $(288,8 \pm 59,0$ versus $245,3 \pm 54,4)$ em comparados aos não expostos. Os níveis médios de creatinina sérica $(\mathrm{mg} / \mathrm{dL})$ também aumentaram significativamente no grupo dos expostos ao benzeno em comparação ao grupo não exposto $(1,1 \pm 0,4$ versus $0,8 \pm 0,2)$. Os níveis séricos de ALP (UI/L) foram significativamente elevados nos indivíduos expostos ao benzeno em comparação com os indivíduos não expostos $(87,3 \pm 22,6$ versus $69,6 \pm 16,5)$. Da mesma forma, indivíduos expostos ao benzeno apresentaram níveis significativamente mais altos de AST e ALT comparado com os não expostos.

Haro-Garcia et al (2012) identificou em seu estudo sobre alterações hematológicas em trabalhadores expostos ao BTX, a macrocitose, que pode estar relacionada a exposição crônica ao benzeno, que produz mudanças estruturais nos componentes celulares da medula óssea, além de seu efeito anaplásico e leucemiogênico.

\section{Genotoxicidade relacionada à exposição ao benzeno}

O benzeno e seus metabólitos podem causar mutagenicidade, clastogenicidade e formar adutos de DNA. Explicações para esse dano causado ao DNA incluem: a formação de adutos de DNA por metabólitos de sua biotransformação; o dano oxidativo; o efeito aneugênico (defeito no fuso mitótico, e consequente não separação dos cromossomos) e clastogênicos (quebra nos mesmos), além do efeito nocivo a topoisomerase II, enzima que desempenha papel importante nos processos de replicação e empacotamento de DNA (Campos et al., 2017).

No estudo de Maciel et al (2019) foi constatado aumento da quantidade de micronúcleos no grupo de frentistas expostos em relação ao grupo controle, o que sugere que as substâncias com potencial genotóxico presentes na gasolina como os BTEX, contribuem para o achado de maior quantidade de micronúcleos nesses profissionais. Salem (2018) constatou em 
seu estudo que os trabalhadores de postos de gasolina apresentaram frequência significativamente maior de fragmentação de DNA, micronúcleos e células mortas nos leucócitos do sangue periférico, comparados aos controles. Ambos os estudos consideraram o consumo de álcool como um fator de confundimento para a apresentação de alterações genotóxicas.

Campos et al (2017) utilizaram o teste cometa para avaliar danos no DNA de frentistas e analistas de combustíveis comparados a pessoas não expostas ao benzeno. Os resultados evidenciaram que os indivíduos expostos possuem grau significativamente maior de danos ao DNA em linfócitos periféricos quando comparados aos de indivíduos não expostos, o que aponta precocemente os efeitos nocivos da exposição, podendo ser considerado um biomarcador de efeito com boa sensibilidade.

Amaral et al (2017) aplicaram o teste cometa para avaliar genotoxicidade em trabalhadores de postos de combustíveis. Os resultados mostraram que os danos genéticos foram maiores em posts que possuíam concentrações menores de benzeno no ar. Este fato indica a existência de uma segunda via de metabolização do benzeno e da influência com a curva dose-resposta em casos de exposição a doses baixas, o que facilita a metabolização e produção de metabólitos tóxicos. Isso indica o risco de danos à saúde mesmo com exposição a doses pequenas de benzeno.

Campos (2013) utilizou o teste cometa para avaliar a genotoxicidade em frentistas e analistas de combustíveis. Houve diferença significativa ao comparar os indivíduos expostos com o grupo controle não exposto. Não foi encontrada correlação significativa da expressão do biomarcador com consumo de álcool e tempo de exposição no grupo de indivíduos expostos. Foi encontrada diferença no índice de dano ao comparar a função dos trabalhadores, com índices mais elevados em frentistas que em analistas de combustíveis.

As manifestações de imunotoxicidade após a exposição crônica ao benzeno estão relacionadas diretamente às alterações na produção de leucócitos e indiretamente aos efeitos que provocam na imunidade que as pessoas podem adquirir através da produção de anticorpos (Giardini, 2016). Ramadan et al (2000), trataram das consequências imunotóxicas da exposição ocupacional de 60 trabalhadores frentistas, não fumantes, de postos de combustíveis. Foram dois grupos de estudo sendo 30 expostos à gasolina por um período entre três e cinco anos e 30 trabalhadores expostos por mais de cinco anos. $\mathrm{O}$ estudo permitiu concluir que houve um aumento no risco de imunotoxicidade e genotoxicidade nos trabalhadores expostos a este combustível, risco esse proporcional ao tempo de exposição à gasolina.

\section{Legislação da exposição ocupacional ao benzeno}

A Norma Regulamentadora 7 da Secretaria Especial de Previdência e Trabalho estabeleceu uma periodicidade para a realização de exames de monitoramento para trabalhadores expostos ou potencialmente expostos a diversas substâncias, dentre elas o benzeno, e a Norma Regulamentadora 9 define os parâmetros para mensuração ambiental (Secretaria Especial de Previdência e Trabalho, 2019).

Uma das principais fontes de exposição ao benzeno em ambiente ocupacional é a gasolina, visto que este hidrocarboneto pode estar presente em volume de 1 a $5 \%$ neste combustível. Em países norte-americanos e europeus, os esforços têm caminhado para que o volume de benzeno na gasolina seja menor que 1\%. A ANP determinou em 2013 que, no Brasil, o volume máximo de benzeno na gasolina tipo C seja de $1 \%$. Considerando-se que a gasolina é um produto de venda livre e tem diversas aplicabilidades nas áreas residenciais, industrial e oficinas mecânicas como solvente de limpeza, o risco de exposição torna-se bastante abrangente (Campos et al., 2017; Mendes et al., 2017).

As leis específicas sobre o benzeno no Brasil são: a Portaria no 14/95, a Instrução Normativa $n^{\circ} 1 / 95$ que dispõe sobre "Avaliação das concentrações de benzeno em ambientes de trabalho" e a Instrução Normativa no 2/95 que dispõe sobre a "Vigilância da saúde dos trabalhadores na prevenção da exposição ocupacional ao benzeno". A Portaria n 776 de 28 de abril de 2004, dispõe sobre a regulamentação dos procedimentos relativos à vigilância da saúde dos trabalhadores expostos ao 
benzeno, e dá outras providências. A NR 15 proíbe o uso de benzeno a partir do ano de 1997 em qualquer ocupação, exceto nos setores industriais e em laboratórios que o produzem ou o utilizam com outras finalidades nas quais não é possível substituí-lo (Brasil, 2011; Confederação Nacional da Indústria, 2012).

O anexo 13-A da NR 15 da Portaria n ${ }^{\circ} 14$ de 1995 estabeleceu o Valor de Referência Tecnológico - Média Ponderada pelo Tempo (VRT-MPT) que, diferente do limite de tolerância, não exclui os riscos a saúde dos expostos. Define-se como VRT-MPT, a concentração média de benzeno no ar ponderado pelo tempo, considerando-se uma jornada de trabalho de 8 horas diárias, na zona de respiração dos trabalhadores. O valore preconizado para VRT-MPT é de 2,5 ppm para indústrias siderúrgicas e 1 ppm para indústrias química, petroquímica e de petróleo.

De acordo com os dados do Cadastro Geral de Empregados e Desempregados (CAGED), no Brasil, em agosto de 2018, havia 39.712 Postos Revendedores de Combustíveis- PRC com um ou mais empregados, contabilizando-se o total de 373.206 empregados neste ramo de atividade. O uso de biomarcadores de exposição ocupacional ao benzeno, tanto hematológicos e bioquímicos quanto genotóxicos, permitem avaliar a presença deste agente tóxico no organismo destes trabalhadores e com isso identificar possíveis danos a saúde dos mesmos (Moreira \& Gomes, 2011; Conceição et al., 2019).

\section{Conclusão}

O benzeno é um composto extremamente tóxico ao ser humano, ocasionando diversos prejuízos à saúde principalmente os relacionados ao sistema hematopoiético. Indivíduos expostos a longo prazo, como os trabalhadores de postos de gasolina, tem maiores riscos de desenvolver patologias graves como neoplasias e danos permanentes em diversos órgãos. $\mathrm{O}$ Brasil deve repensar sua política de exposição ocupacional ao benzeno tendo em vista seus inúmeros efeitos deletérios. É necessário pensar no impacto econômico a ser gerado pela extinção desta profissão, visto que o país já possui altas taxas de desemprego. A legislação brasileira que dispõe sobre a exposição ao benzeno em ambiente ocupacional precisa incluir o fornecimento de paramentação obrigatória para estes profissionais. Através desta revisão, constatou-se que os valores para monitoramento da exposição ocupacional ao benzeno precisam ser revisados, visto que há décadas não são modificados e podem não contemplar mais a realidade da exposição ao benzeno atualmente. São necessários mais estudos sobre monitoramento da exposição ao benzeno em trabalhadores expostos por longos períodos e os efeitos sobre a saúde a longo prazo.

\section{Referências}

Amaral, I. C. C., Carvalho, L. V. B., Pimentel, J. N. S., Pereira, A. C., Vieira, J. A., Castro, V. S., Borges, R. M., Alves, S. R., Nogueira, S. S., Tabalipa, M. M., Otero, U. B., Oliveira, K. M. P. G., Correa, S. M., Fonseca, A. S. A., Moreira, J. C., Peres, F., Teixeira, L. R., Menezes, M. A. C., Mattos, R. C. O. C., Sarcinelli, P. N. \& Larentis, A. L. (2017). Avaliação ambiental de BTEX (benzeno, tolueno, etilbenzeno, xilenos) e biomarcadores de genotoxicidade em trabalhadores de postos de combustíveis. Revista Brasileira de Saúde Ocupacional, 42(supl 1):e8s.

Araújo, E. C. (2015). Níveis de trans, trans-mucônico na urina como biomarcador de exposição ao benzeno e alteraçães hematológicas na população do bairro Piquiá de Cima, Açailândia-MA. Dissertação de mestrado, Escola Nacional de Saúde Pública Sergio Arouca, Rio de Janeiro.

Arcuri, A., Costa, A., Machado, J., Cardoso, L., Magrini, R. \& Marra, V. (2005). Vigilância do risco químico módulo do Benzenismo. Versão para consulta pública. Área técnica de Saúde do Trabalhador, Ministério da Saúde, 1-38.

Brasil. Ministério do Trabalho e Emprego. (2011). Portaria n 3.214, Norma Regulamentadora 15, Anexo nº 13, Agentes químicos. Brasília, 8 de Junho de 1978. Alterada pela Portaria n 291 de 8 de dezembro de 2011.

Brasil. Ministério do Trabalho e Emprego. (1995). Secretaria de Segurança e Saúde no Trabalho. Portaria no 14 de 20 de Dezembro de 1995.

Campos, M. A. A. (2013). Teste Cometa: validação do método e avaliação da exposição ocupacional ao benzeno presente na gasolina através dos biomarcadores de exposição e genotoxicidade. Dissertação de mestrado, Universidade Federal de Minas Gerais.

Campos, M. A. A., Fernandes, A. P. S. M. \& André, L. C. (2017). Avaliação da exposição ocupacional ao benzeno em trabalhadores frentistas e analistas de combustíveis utilizando o Teste Cometa como biomarcador de genotoxicidade. Revista Brasileira de Saúde Ocupacional, 42(supl 1):e6s. 
Carrieri, M., Pigini, D., Martinelle, A., Paci, E., Maratini, F., Salamon, F. \& Tranfo, G. (2019). Effect of Benzene Exposure on the Urinary Biomarkers of Nucleic Acid Oxidation in Two Cohorts of Gasoline Pump Attendants. International Journal of Environmental research and Public Health, $16,129$.

Chaiklieng, S., Suggaravetsiri, P., Kaminski, N. \& Autrup, H. (2019). Factors Affecting Urinary tt-Muconic Acid Detection among Benzene Exposed Workers at Gasoline Stations. International Journal of Environmental Research and Public Health, 16, 4209.

Cintra, DS (2016). Estudo dos efeitos hematológicos e bioquímicos em trabalhadores de postos de revenda de combustiveis expostos a gasolina no ambiente laboral no município do Rio de Janeiro. Dissertação de mestrado, Fundação Oswaldo Cruz.

Conceição, O. S. A., Cavalcanti, V. L., Brandão, J. S. \& Ramos, M. F. (2019) Prevenção da exposição ocupacional ao benzeno em postos revendedores de combustíveis: atuação da auditoria fiscal do trabalho na Bahia. Revista Labore, 2, 11-35.

Confederação Nacional da Indústria. (2012). Consolidação da legislação sobre o benzeno. Recuperado em outubro de 2020, de http://www.revistaemergencia.com.br/upload/protecao_materiaarquivo/495.pdf

Corrêa, M. J. M. (2014) Prevalência da exposição ocupacional ao benzeno e mortalidade por leucemia entre expostos: estimativas para o Brasil. Tese de doutorado, Instituto de Saúde Coletiva. Universidade Federal da Bahia.

Correa, M. J. M \& Santana, V. S. (2016). Exposição ocupacional ao benzeno no Brasil: estimativas baseadas em uma matriz de exposição ocupacional. Caderno de Saúde Pública, 32(12):e00129415.

Costa-Amaral, I. C. (2017). Estudo da exposição ocupacional e ambiental ao benzeno em baixas concentrações sob a perspectiva dos efeitos genotóxicos. Tese de doutorado, Escola Nacional de Saúde Pública Sergio Arouca, Rio de Janeiro.

Costa-Amaral, I. C., Carvalho, L. V. B., Santos, M. V. C., Valente, D., Pereira, A. C., Figueiredo, V. O., Souza, J. M., Castro, V. S., Trancoso, M. F., Fonseca, A. S. A., Milagres, V. G., Mendes, M. P. R., Paiva, M. J. N., André, L. C., Borges, R. M., Menezes, M. A. C., Alves, S. R., Gonçalves, . E. S., Sisenando, H. A., Perini, J. A., Oliveira, M. S., Moura-Correa, M. J., Teixeira, L. R., Collins, A. R., Mattos, R. C. O. C., Sarcinelli, P. N. \& Larentis, A. L. (2019). Environmental Assessment and Evaluation of Oxidative Stress and Genotoxicity Biomarkers Related to Chronic Occupational Exposure to Benzene. International Journal of Environmental Research and Public Health, 25;16(12):2240.

D’alascio, R. G., Menegali, M., Bornelli, A. S. \& Magajewski, F. (2014). Sintomas relacionados à exposição ocupacional ao benzeno e hábitos ocupacionais em trabalhadores de postos de revenda de combustíveis a varejo na região sul de Santa Catarina. Revista Brasileira de Medicina do Trabalho, 12(1):21-9.

D'andrea, M. A. \& Reddy, G. K. . (2014). Hematological and hepatic alterations in nonsmoking residents exposed to benzene following a flaring incident at the British petroleum plant in Texas City. Environmental health, 13:115. 10.1186/1476-069X-13-115

D'andrea, M. A. \& Reddy, G. K. (2014). Adverse Health Effects of Benzene Exposure Among Children Following a Flaring Incident at the British Petroleum Refinery in Texas City. Pediatric Hematology and Oncology, 31: 1, 1-10. 10.3109/08880018.2013.831511

Figueiredo, V. O. (2018). Avaliação da exposição ao benzeno, tolueno, etilbenzeno e xilenos (BTEX) em trabalhadores expostos ocupacionalmente, no município do Rio de Janeiro. Dissertação de Mestrado, Fundação Oswaldo Cruz, Escola Nacional de Saúde Pública Sergio Arouca, Rio de Janeiro.

Fonseca, A. S. A., Costa, D. F., Dapper, V., Machado, J. M. H., Valente, D., Carvalho, L. V. B., Costa-Amaral, I. C., Alves, S. R., Sarcielli, P. N., Menezes, M. A. C., Larentis, A. L., Moura-Correa, M. J. \& Mattos, R. C. O. (2017). Classificação clínico-laboratorial para manejo clínico de trabalhadores expostos ao benzeno em postos de revenda de combustíveis. Revista Brasileira de Saúde Ocupacional, 42(supl 1):e5s.

Forsell, K.., Liljelind, I., Ljungkvist, G., Nordlinder, R., Andersson, E. \& Nilsson, R. (2019). Benzene Exposure and Biomarkers in Alveolar Air and Urine Among Deck Crews on Tankers Transporting Gasoline. Annals of Work Exposures and Health, 63, 8, 890-897.

Giardini, I. (2016). Avaliação dos efeitos genotóxicos associados a exposição ao benzeno em trabalhadores de postos de combustíveis no município do Rio de Janeiro. Dissertação de mestrado, Instituto Nacional de Controle de Qualidade em Saúde, Fundação Oswaldo Cruz.

Gonçalves, E. S., Borges, R. M., Carvalho, L. V. B., Alves, S. R., André, L. C. \& Moreira, J. C. (2017). Estratégias analíticas com cromatografia e espectrometria de massas para biomonitorização da exposição ao benzeno pela determinação do ácido S-fenilmercaptúrico urinário. Revista Brasileira de Saúde Ocupacional, 42(supl 1):e1s.

Haro-García, L., Vélez-Zamora, N., Aguilar-Madriz, G., Guerrero-Rivera, S., Sánchez-Escalante, V., Muñoz, Sr., Mezones-Holguín, E. \& Juárez-Pérez, C. (2012). Alteraciones hematológicas en trabajadores expuestos ocupacionalmente a mezcla de Benceno-Tolueno-Xileno (BTX) en una fábrica de pinturas. Revista Peruana de Medicina Experimental y Salud Pública, 29(2):181-87.

Ibrahim, K. S., Amer, N. M., El-Dossuky, E. A., Emara, A. M., El-Fattah , A. \& Shahy, E. M. (2014). Hematological effect of benzene exposure with emphasis of muconic acid as biomarker. Toxicology and Industrial Health, 30 (5): 467-474. 10.1177 / 0748233712458141

Ketan, V. K., Bhavyata, K., Linzbuoy, G. \& Hyacinth, H. N. (2015). Renal and hepatotoxic alterations in adult mice on inhalation of specific mixture of organic solvents. Toxicology and Industrial Health, 31 (12): 1158-1164. 10.1177 / 0748233713485892

Khalade, A., Jaakkola, M. S., Pukkala, E. \& Jaakkola, J. J. (2010). Exposure to benzene at work and the risk of leukemia: a systematic review and metaanalysis. Environmental Health, 28;9:31. 10.1186/1476-069X-9-31

Kim, J. H., Moon, J. Y., Park, E. Y., Lee, K. H. \& Hong, Y. C. (2011). Changes in Oxidative Stress Biomarker and Gene Expression Levels in Workers Exposed to Volatile Organic Compounds. Industrial Health, 49, 8-14.

Landim, A. C. F., Pinheiro, F. M., Pessanha, F. S., Santos, L. \& Valente, G. S. C. (2015). Assistência de enfermagem a idosos com traumas ósseos: uma revisão integrativa. Revista de Pesquisa Cuidado é Fundamental Online. 7(1): 2083-2103. 10.9789/2175-5361.2015.v7i1.2083-2103

Li, P., Wu,Y., Zhang, Z., Lin, D., Wang, D., Huang, X. \& Zhang, Y. (2019). Proteomics analysis identified serum biomarkers for occupational benzene exposure and chronic benzene poisoning. Medicine, 98:25. 
Maciel, L. A., Feitosa, S. B., Trolly, T. S. \& Sousa, A. L.(2019). Avaliação dos efeitos genotóxicos da exposição ocupacional em frentistas atuantes em postos de gasolina no município de Santarém, Pará. Revista Brasileira de Medicina do Trabalho, 17(2):247-5.

Manini, P., De Palma, G., Andreoli, R., Poli, D., Mozzoni, P., Folesani, G., Mutti, A. \& Apostoli. P. (2007). Environmental and biological monitoring of benzene exposure in a cohort of Italian taxi drivers. Toxicology Letters. 167(2), 142-51. 10.1016/ j.toxlet.2006.08.016

Pelallo-Martínez, N. A., Batres-Esquivel, L., Carrizales-Yáñez, L., \& Díaz-Barriga, F. M. (2014). Genotoxic and hematological effects in children exposed to a chemical mixture in a petrochemical area in Mexico. Archives of environmental contamination and toxicology, 67(1), 1-8. 10.1007/s00244-014-9999-4

Mendes, K. D. S., Silveira, R. C. C. P. \& Galvão, C. M. (2008). Revisão integrativa: método de pesquisa para a incorporação de evidências na saúde e na enfermagem. Texto \& Contexto - Enfermagem. 17(4): 758-64. 10.1590/S0104-07072008000400018

Mendes, M., Machado, J. M. H., Durand, A., Costa-Amaral, I. C., Valente, D. \& Gonçalves, E. S. (2017). Normas ocupacionais do benzeno: uma abordagem sobre o risco e exposição nos postos de revenda de combustíveis. Revista Brasileira de Saúde Ocupacional; 42 (Supl. 1):1-19.

Moreira, G. M. \& Gomes, S. F.(2011). Intoxicação ocupacional pelo benzeno: um assunto de saúde ambiental. Revista Brasileira de Odontologia, 68(2) 171-4.

Moro, A. M., Sauer, E., Brucker, N., Charão, M. F., Gauer, B., Nascimento, S. N., Goethel, G., Duarte, M. M. M. F. \& Garcia, S. C. (2019). Evaluation of immunological, inflammatory, and oxidative stress biomarkers in gasoline station attendants. BMC Pharmacology and Toxicology, 20 (Suppl 1):75.

Moura-Correa, M. J., Jacobina, A. J. R., Santos, A. S., Pinheiro, R. C., Menezes, M. A. C., Tavares, A. M. \& Pinto, N. F. (2014). Exposição ao benzeno em postos de revenda de combustíveis no Brasil: Rede de Vigilância em Saúde do Trabalhador (VISAT). Revista Ciência e Saúde Coletiva [online]. 19, 12, 46374648. 10.1590/1413-812320141912.12772014

Nogueira, S. M. (2016). Polimorfismos metabólicos e alterações clínicas e laboratoriais relacionadas à exposição ao benzeno em trabalhadores de postos de combustíveis da cidade do Rio de Janeiro. (Tese de doutorado) - Escola Nacional de Saúde Pública Sergio Arouca, Rio de Janeiro.

Ramadan, M. M., Osman, H. S. \& Moussa, M. E. K.(2000). Chronic exposure to gasoline: evaluation of immunological and genotoxic effects among filling station workers. Mansoura Journal of Forensic Medicine and Clinical Toxicology. 8, 2, 85-98.

Ray, M. R., Roychoudhury, S., Mukherjee, S., \& Lahiri, T. (2007). Occupational benzene exposure from vehicular sources in India and its effect on hematology, lymphocyte subsets and platelet P-selectin expression. Toxicology and industrial health, 23(3), 167-175. 10.1177/0748233707080907

Ruiz, M. A., Augusto, L. G., Vassallo, J., Vigorito, A. C., Lorand-Metze, I., \& Souza, C. A. (1994). Bone marrow morphology in patients with neutropenia due to chronic exposure to organic solvents (benzene): early lesions. Pathology, research and practice, 190(2), 151-154. 10.1016/S0344-0338(11)80705-3

Salem, E., El-Garawani, I., Allam, H., El-Aal, B. \& Hegazy, M. (2018). Genotoxic effects of occupational exposure to benzene in gasoline station workers. Industrial Health. 56(2):132-40. 10.2486/indhealth.2017-0126

Santos, M. V. C., Figueiredo, V. O., Arcuri, A. S. A., Costa-Amaral, I. C., Gonçalves, E. S. \& Larentis, A. L. (2017). Aspectos toxicológicos do benzeno, biomarcadores de exposição e conflitos de interesses. Revista Brasileira de Saúde Ocupacional. 42 (supl 1):e13s. 20.

Swaen, G. M., Van Amelsvoort, L., Twisk, J. J., Verstraeten, E., Slootweg, R., Collins, J. J. \& Burns, C. J. (2010). Low level occupational benzene exposure and hematological parameters. Chemico-biological interactions, 184(1-2), 94-100. 10.1016/j.cbi.2010.01.007

Valente, D., Costa-Amaral, I. S., Carvalho, L. V. B., Santos, M. V. C., Castro, V. S., Rodrigues, D. R. F, Falco, A., Silva, C. B., Nogueira, S. M., Gonçalves, E. S., Moreira, J. C., André, L. C., Teixeira, L. R., Sarcinelli, P. N., Sisenando, H. A., Oliveira, M. S., Perini, J. A., Mattos, R. C. O. C. \& Larentis, A. L. (2017). Utilização de biomarcadores de genotoxicidade e expressão gênica na avaliação de trabalhadores de postos de combustíveis expostos a vapores de gasolina. Revista Brasileira de Saúde Ocupacionais [online]. 42(supl 1):e2s. DOI:10.1590/2317-6369000124415

Weisel, C. P. (2010). Benzene exposure: an overview of monitoring methods and their findings. Chemico-Biological Interactions, 184(1-2):58-66. 10.1016/j.cbi.2009.12.030 